\title{
Lobes de stabilité en UGV approche expérimentale en usinage de poches
}

\author{
Nicolas Blanchard ${ }^{a}$, Thierry Rabany et Emmanuel Duc \\ IFMA, BP 265, 63175 Aubière Cedex, France
}

Reçu le 14 février 2005, accepté le 13 avril 2005

\begin{abstract}
Résumé - La théorie des lobes de stabilité en UGV est aujourd'hui maîtrisée pour des usinages suivant des trajectoires rectilignes et à engagement radial constant. Pour des trajets contenant des arcs de cercles, le diagramme des lobes de stabilité en trajectoire rectiligne n'est plus applicable directement. L'étude expérimentale qui suit propose différentes options et réglages permettant d'utiliser les lobes de stabilité en usinage rectiligne dans le cadre de l'usinage de poches. Les options de programmation de la commande numérique, la consigne de vitesse d'avance et l'engagement radial sont plus particulièrement étudiés et optimisés pour obtenir des conditions les plus stables possibles.
\end{abstract}

Mots clés : Lobes de stabilité / usinage grande vitesse / usinage de poches / vibrations

\begin{abstract}
Stability charts for high speed milling experimental study of pocket milling. Nowadays, the stability charts applied to high speed milling are in use for chatter prediction in linear trajectories with constant radial depth of cut. When it comes to all other moves, including circular paths, the charts used for linear movements are not always valid. The following experimental study explores the parameters and adjustments allowing the extension of the use of the stability charts for the linear cases, to the pocket milling cases. The NC advanced programming options, the feed rate and the radial depth of cut are studied in detail and optimized using milling stability as a criterion.
\end{abstract}

Key words: Stability charts / high speed milling / pocket milling / vibrations / chatter

\section{Introduction}

L'utilisation de l'usinage grande vitesse permet souvent des gains importants dans l'enlèvement de matière. L'apparition de vibrations pendant l'usinage peut cependant limiter sa mise en œuvre dans un contexte industriel. Ces vibrations limitent le débit copeau, détériorent la qualité de la pièce, et peuvent conduire à une ruine accélérée de l'outil et de la machine.

Pour éviter leur apparition, plusieurs types de réponses ont été apportés. Elles incluent le développement d'outils, ou de porte-outils plus rigides, ou ayant des propriétés d'amortissement [1] et des études plus détaillées du phénomène de vibration [2]. Les causes d'apparition de la vibration sont aujourd'hui connues : parmi les principales citons le frottement de l'outil sur la pièce, les actions thermodynamiques de la coupe, le couplage des modes, et enfin, la théorie la plus répandue, celle de la vibration

\footnotetext{
a Auteur pour correspondance:
}

nicolas.blanchard@ifma.fr auto-régénérative développée par Altintas [3,4]. De nombreux modèles théoriques pour le fraisage ont été élaborés en vue de la prédiction de ces vibrations. Engin et Altintas [4] ont travaillé sur des modèles analytiques à deux degrés de liberté avec un comportement dynamique du système vibrant et de la coupe indépendant des vitesses de rotation de broche. Faassen [5] a essayé un modèle dépendant de la rotation et paramétré. Assouline [6] a étudié un modèle numérique et Insperger [7] un modèle à un seul degré de liberté. La plupart de ces travaux ne portent que sur des usinages à engagements radiaux et axiaux constants sur des trajectoires rectilignes mis à part quelques études, comme celle de Bayly sur l'immersion radiale [8].

La limite entre usinage stable et instable, est représentée par le diagramme des lobes de stabilité sur un graphique, avec en abscisse la vitesse de rotation de la broche, et en ordonnée la profondeur de passe [9]. L'étude expérimentale présentée ici s'intéresse au comportement vibratoire en usinage de poches. Le diagramme (théorique ou expérimental) des lobes de stabilité dans des conditions 
d'usinage rectiligne données, peut être utilisé pour l'usinage de poches à fonds plats. Pour cela, certaines règles doivent être appliquées, permettant à l'usinage de rester stable, en particulier dans les passages d'angles. Un état des lieux des conditions affectant l'apparition de la vibration est d'abord posé. Quelques règles préalables de programmation de la commande numérique sont déduites d'une première analyse. Le protocole expérimental de tracé du diagramme des lobes de stabilité est ensuite détaillé. Les résultats sont présentés puis discutés, et des conditions d'utilisation des diagrammes de lobes de stabilité en usinage de poche sont proposées.

\section{Conditions d'apparition de la vibration}

Les nombreuses études théoriques citées admettent quasiment toutes que le diagramme des lobes de stabilité, et donc la limite de vibration en usinage rectiligne, est fonction des paramètres suivants :

- Le nombre de dents de l'outil : $Z$;

- les coefficients spécifiques de coupe tangentiel et radial : $K_{\mathrm{t}}$ et $K_{\mathrm{r}}$;

- les conditions d'immersion de l'outil (angles d'entrée et de sortie de la matière) : $\Phi_{\mathrm{e}}$ et $\Phi_{\mathrm{S}}$;

- les paramètres du comportement dynamique de l'ensemble vibrant (machine/broche/porte-outil/outil) : la pulsation propre $\omega_{\mathrm{p}}$, l'amortissement $\xi$ et la rigidité $k$.

Entre un usinage rectiligne et un usinage de poche, les paramètres du comportement dynamique de la machine $\omega_{\mathrm{p}}$, $\xi$ et $k$, et le nombre de dents de l'outil $Z$ restent invariables tant que le système expérimental (machine/attachement/outil/matière/montage) est inchangé. Par contre, $K_{\mathrm{t}}$ et $K_{\mathrm{r}}$ varient avec $F_{z}$ (l'avance à la dent) ainsi que les angles $\Phi_{\mathrm{e}}$ et $\Phi_{\mathrm{s}}[3,4,7]$. Les conditions qui varient dans les rayons de courbure se limitent donc à $\Phi_{\mathrm{e}}, \Phi_{\mathrm{s}}, F_{z}$ et $A_{\mathrm{e}}$, engagement radial, lié directement à $\Phi_{\mathrm{e}}$ et $\Phi_{\mathrm{s}}$.

\section{Programmation de la commande numérique}

La commande numérique doit être programmée de manière à garder la vitesse d'avance réelle la plus proche de la vitesse de consigne. Du fait de la dynamique de la machine, plusieurs paramètres d'usinage, supposés constants dans l'application de la théorie des lobes de stabilité [3] varient pendant le parcours du trajet d'usinage. Pour continuer à utiliser les diagrammes de lobes de stabilité construits en usinage rectiligne sur une trajectoire plus complexe, il faut être le plus souvent possible à la vitesse de consigne, et limiter ainsi la variation de $F_{z}$.

À chaque instant, la vitesse instantanée dépend de la capacité d'accélération de la machine, du JERK, et du traitement de la commande numérique [10]. Deux essais avec des valeurs de JERK à $50 \%$ et à $100 \%$ montrent que

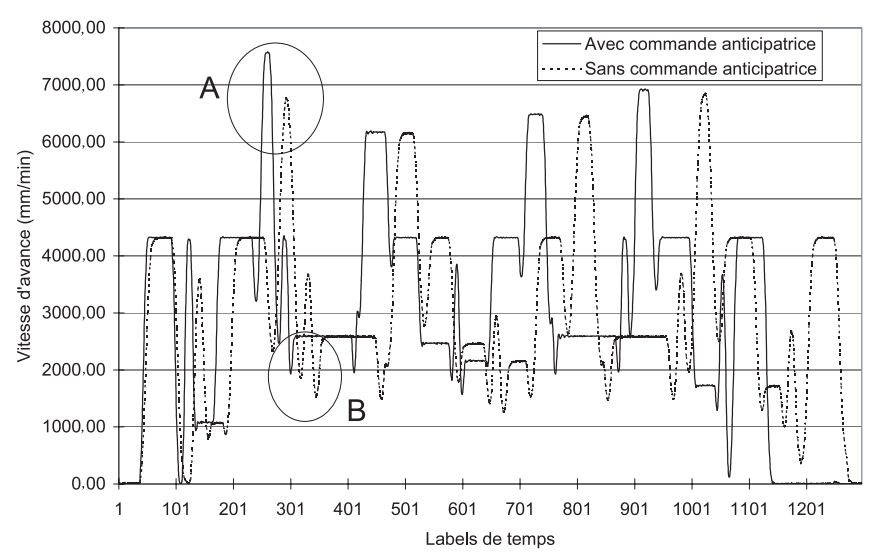

Fig. 1. Influence de la commande d'anticipation de trajectoire.

la capacité de la machine à atteindre sa vitesse de consigne n'est pas affectée par ce réglage pour la trajectoire d'essai. Le JERK est donc réglé à $50 \%$ pour éviter des chocs dans les actionneurs lors des déplacements.

D'autre part, l'anticipation de trajectoire [11] (FFWON) qui annule l'erreur de poursuite permet de rester plus souvent à la consigne de vitesse, spécialement dans les raccordements entre les différentes portions de trajectoires rectilignes et circulaires comme dans les zones A et B sur la figure 1. La commande anticipatrice est donc utilisée pour l'étude.

La troisième option à étudier, est le paramétrage du contournage à la transition entre deux blocs de programmation (raccordement entre deux portions de trajectoire). La machine ralentit au passage d'une transition pour suivre le profil de consigne avec une certaine erreur [11]. Deux possibilités existent alors : un contournage par défaut G64, pré-paramétré en fonction de la vitesse d'avance, ou un contournage G642 paramétrable par l'utilisateur avec une tolérance donnée. Selon l'option choisie et son paramétrage, la machine va plus vite, ou est plus précise dans le passage de la zone de transition.

La figure 2 montre deux essais faits sur le même trajet de mesure que précédemment, l'un avec le contournage par défaut G64, et l'autre avec le contournage G642 paramétré avec une tolérance d'usinage de 0,02 $\mathrm{mm}$. Cette seconde option est retenue car elle permet de rester plus souvent à la vitesse de consigne, spécialement dans la zone $\mathrm{A}$ où la vitesse du mobile est importante. Les zones B et $\mathrm{C}$ montrent le ralentissement plus important de G64 même pour des vitesses d'avance peu élevées. À la vitesse d'avance maximale, G642 permet d'être à la vitesse de consigne pendant $73 \%$ du temps de parcours, contre seulement $45 \%$ pour G64.

Pour la suite de l'étude, les usinages s'effectuent donc en utilisant un JERK réglé à $50 \%$ de la valeur nominale, l'anticipation de trajectoire FFWON, et une option de contournage G642 paramétrée à $0,02 \mathrm{~mm}$. La variable d'avance à la dent $F_{z}$ est ainsi conservée la plus constante possible, proche des conditions d'un usinage rectiligne. Le diagramme des lobes de stabilité correspondant à l'usinage de la poche avec ces réglages doit donc être peu 


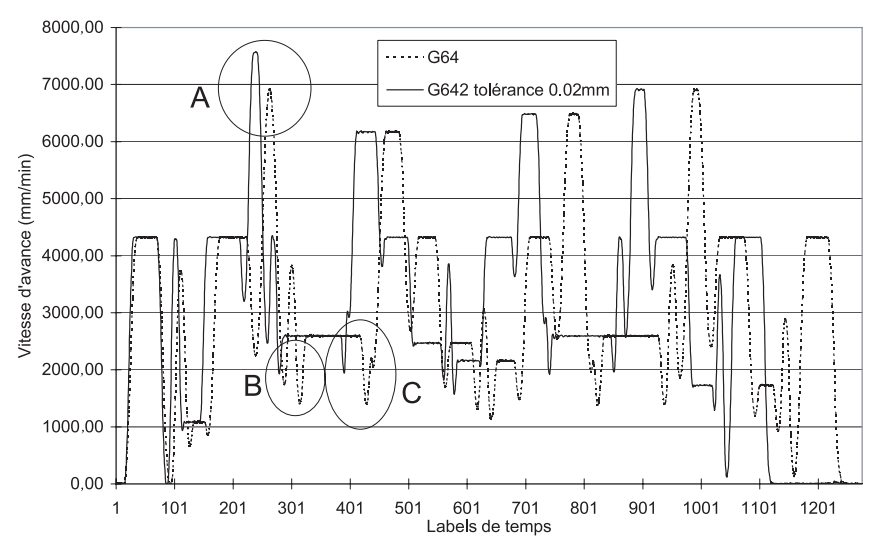

Fig. 2. Influence du choix G64/G642 avec une tolérance d'usinage de $0,02 \mathrm{~mm}$.

déformé par rapport à celui obtenu en trajectoire rectiligne.

\section{Dispositif expérimental}

La matière choisie pour les essais est un alliage d'aluminium 7075 utilisé en aéronautique sous la dénomination de FORTAL HR (Pechiney Rhenalu). L'outil sélectionné est une fraise torique de diamètre $12 \mathrm{~mm}$ et de rayon en bout 1,5 $\mathrm{mm}$. Elle possède une longueur détalonnée de $51 \mathrm{~mm}$, et une longueur totale de $97 \mathrm{~mm}$. Elle a été choisie non revêtue pour avoir une meilleure acuité d'arête, elle est de marque FRAISA, référence 5277.501. Elle est montée dans un attachement EPB HSK-63A en pince équilibrée type D-24.

La machine utilisée pour tous les essais est le centre d'usinage grande vitesse Huron KX-15 présent à l'IFMA équipé d'une commande numérique Siemens 840D. Il possède une broche de taux de rotation maximal de 18000 tr. $\mathrm{min}^{-1}$.

Les mesures d'efforts sont faites à l'aide d'un dynamomètre Kistler à trois composantes sur lequel est fixé le bloc de matière. La table de mesure elle-même, est vissée directement sur le bâti de la machine. Le dynamomètre est relié à un amplificateur puis à une chaîne d'acquisition permettant de traiter les signaux d'efforts mesurés sur les trois axes $X, Y$ et $Z$. Le comportement vibratoire est déduit des efforts par une transformée de Fourier qui donne la fonction de transfert du signal. La mesure faite sur tout le profil de la poche est découpée par zones. Les parties de transitions entre deux zones sont éliminées. La mesure des efforts de chaque zone du contour est analysée séparément.

La géométrie de poche à fond plat choisie pour l'étude est présentée sur la figure 3. Elle comprend des trajets rectilignes, et des courbures convexes et concaves de rayons $8 \mathrm{~mm}, 10 \mathrm{~mm}, 12 \mathrm{~mm}$ et $14 \mathrm{~mm}$. Les rayons choisis sont volontairement proches du rayon de l'outil, puisque ces conditions sont les plus défavorables du point de vue des vibrations, mais aussi des efforts de coupe et de la maîtrise de la vitesse d'avance.

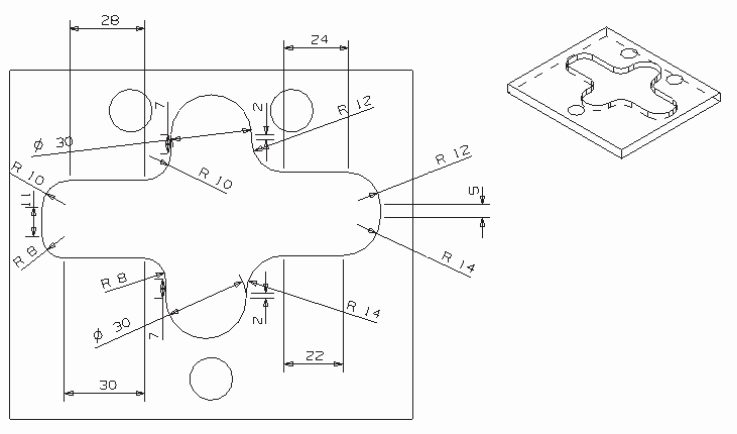

Fig. 3. Schéma de la poche d'étude.

La poche est ouverte en son centre sur une profondeur de $6 \mathrm{~mm}$ en laissant une surépaisseur latérale de $8 \mathrm{~mm}$ sur le profil final. Cette épaisseur est programmée à l'aide d'un logiciel de fabrication assistée par ordinateur (FAO) avec une tolérance d'usinage de 0,001 $\mathrm{mm}$. La mesure des efforts est faite sur la dernière passe le long du profil de la figure 3 en utilisant une combinaison d'interpolation linéaires et circulaires et en tenant compte de la correction de rayon d'outil. La consigne de vitesse d'avance est appliquée à la périphérie de l'outil.

\section{Résultats des essais}

En utilisant tous les résultats précédents, les diagrammes des lobes de stabilité sont tracés pour les rayons concaves de $8 \mathrm{~mm}, 10 \mathrm{~mm}, 12 \mathrm{~mm}$ et $14 \mathrm{~mm}$ de la poche d'étude.

Les résultats portés sur la figure 4 montrent un léger écrasement des diagrammes des lobes de stabilité, et un décalage vers des profondeurs de passe plus faibles au fur et à mesure que les rayons des passages d'angles diminuent. L'ensemble dynamique vibrant machine/porteoutil/outil, n'étant pas modifié, les fréquences de rotation de la broche correspondant aux lobes de stabilité demeurent les mêmes. La diminution de la profondeur de passe maximale avant perte de la stabilité n'est pas uniforme sur tout le diagramme, et oscille entre 1 et $2 \mathrm{~mm}$ selon les points. Il est difficile de dégager une loi simple permettant de quantifier directement cette diminution.

La vitesse d'avance est conforme à la consigne sur les zones de mesure. L'avance à la dent $F_{z}$ est donc identique entre usinage rectiligne et passages d'angles.

Des diagrammes de lobes de stabilité ont été tracés en appliquant la consigne de vitesse, non plus à la périphérie de l'outil, mais en son centre, la vitesse d'avance à la dent $F_{z}$ augmente alors lors du passage des rayons concaves. La comparaison entre les deux diagrammes de lobes est montrée sur la figure 5 pour des rayons de courbure de $8 \mathrm{~mm}$ et $10 \mathrm{~mm}$. Les zones A et B montrent que le fait d'appliquer l'avance à la périphérie de l'outil permet de s'approcher des conditions de stabilité en usinage rectiligne, et de faire remonter la limite de stabilité de $0,5 \mathrm{~mm}$ à $1 \mathrm{~mm}$ à l'intérieur des lobes. 


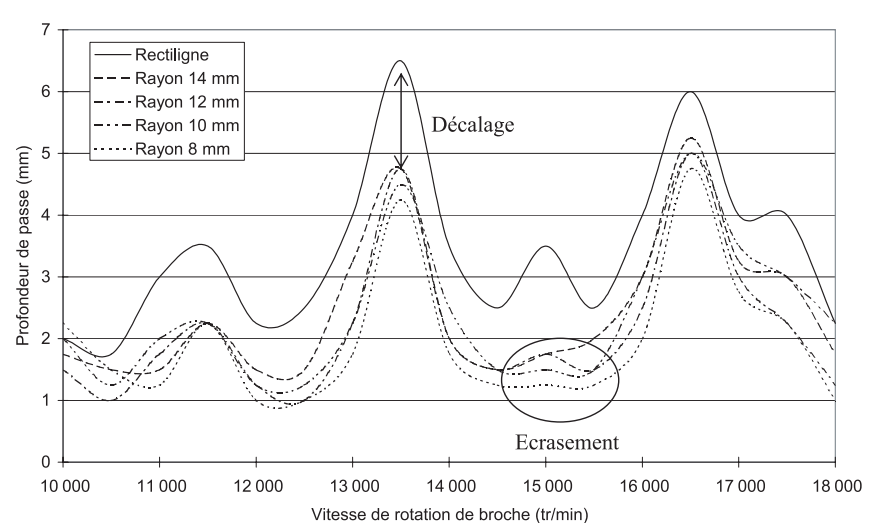

Fig. 4. Diagrammes des lobes de stabilité pour différents rayons de courbure.

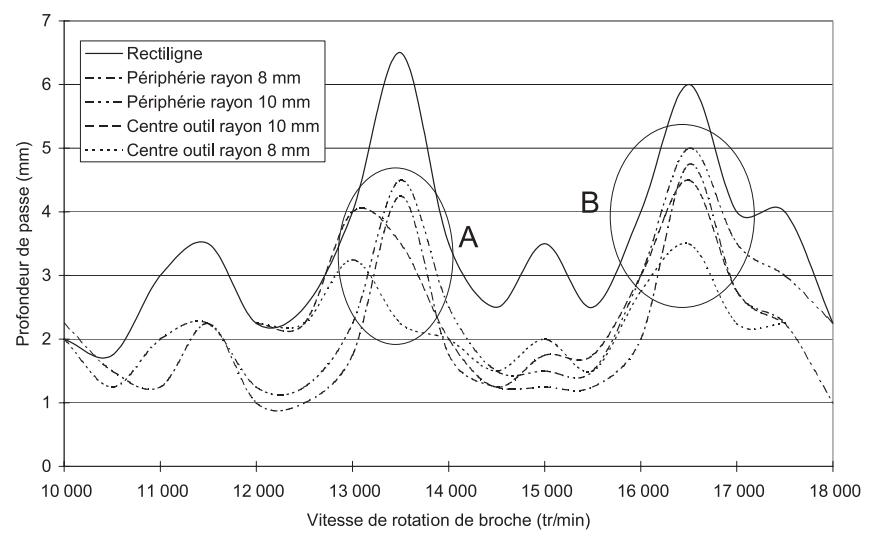

Fig. 5. Influence du pilotage de la vitesse sur le diagramme des lobes de stabilité pour des rayons de courbure de $8 \mathrm{~mm}$ et $10 \mathrm{~mm}$.

La maîtrise de l'avance à la dent $F_{z}$ est une des clés importantes de l'application des diagrammes de lobes de stabilité en usinage rectiligne à l'usinage de poches à fonds plats. Il faut faire un choix correct des options de programmation de la commande numérique : le JERK, l'anticipation de trajectoire, et les options de contournage doivent être réglés pour que l'avance réelle soit le plus souvent égale à la consigne de vitesse tout au long du profil. La consigne d'avance à la dent doit être appliquée à la périphérie de l'outil.

Des mesures ont aussi été faites pour des rayons identiques, mais convexes présents sur la poche d'étude, les lobes se trouvent au-dessus de ceux tracés en usinage rectiligne, ces portions de trajectoires ne limitent pas la stabilité en usinage de poche.

\section{Influence de la variation de l'engagement radial réel}

Une surépaisseur radiale de matière constante de $8 \mathrm{~mm}$ a été programmée en FAO entre le trajet de finition et le trajet de demi-finition. La variation de l'engagement radial $A_{\mathrm{e}}$ affecte directement $\Phi_{\mathrm{e}}$ et $\Phi_{\mathrm{s}}$, et donc la limite de stabilité.

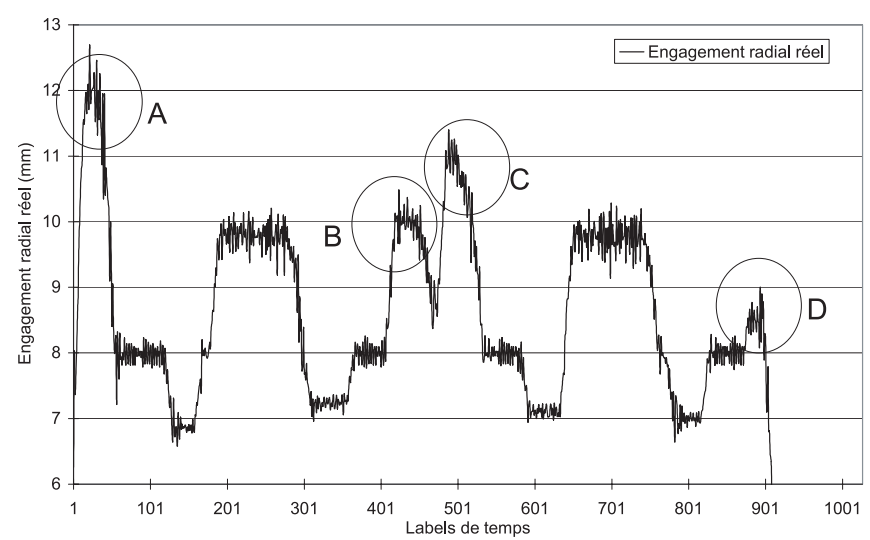

Fig. 6. Variation de l'engagement radial réel le long du profil pour une surépaisseur programmée de $8 \mathrm{~mm}$.

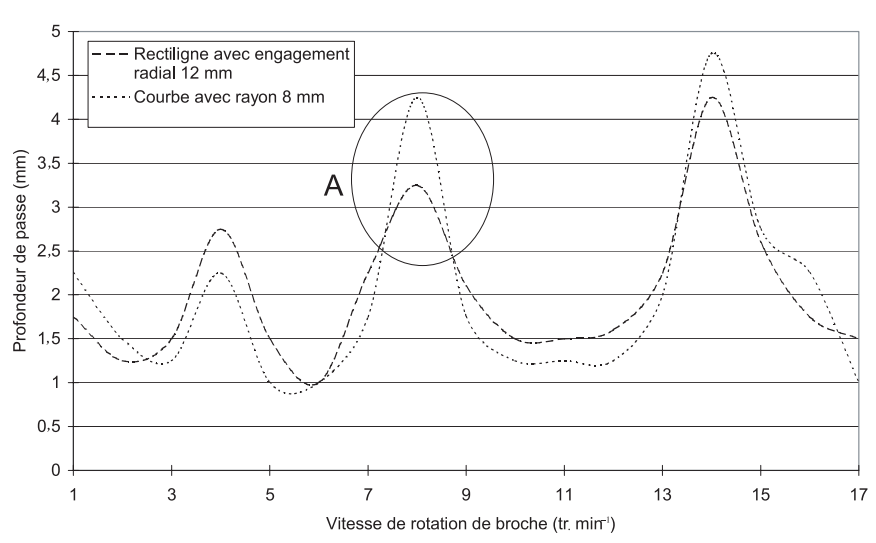

Fig. 7. Comparaison des lobes de stabilité pour une trajectoire rectiligne d'engagement radial de $12 \mathrm{~mm}$ et un rayon de courbure de $8 \mathrm{~mm}$.

La mesure par le calcul de l'engagement radial réel $A_{\mathrm{e}}$, tout au long du profil, est reconstruite sur la figure 6 . $A_{\mathrm{e}}$ n'est pas constant le long du parcours, et varie même de manière importante, malgré les précautions prises au moment de la programmation de la surépaisseur.

La zone A correspond au rayon de $8 \mathrm{~mm}$, la zone $\mathrm{B}$ au rayon de $14 \mathrm{~mm}$, la zone $\mathrm{C}$ au rayon de $12 \mathrm{~mm}$ et la zone D au rayon de $10 \mathrm{~mm}$. Les engagements radiaux réels moyens sont respectivement de 12,0 mm, 10,0 mm, $10,7 \mathrm{~mm}$ et $8,5 \mathrm{~mm}$, pour ces quatre zones. La variation de cet engagement radial, affecte les angles d'entrée et de sortie matière, et donc peut-être la stabilité de l'usinage.

Dans les mêmes conditions que précédemment, des diagrammes de lobes de stabilité en usinage rectiligne ont été tracés pour des valeurs d'engagement radial de $10 \mathrm{~mm}$ et $12 \mathrm{~mm}$. Les figures 7 et 8 montrent ces diagrammes ainsi que ceux précédemment tracés en usinage de poche pour des zones où l'engagement radial réel est identique. L'engagement radial de $10 \mathrm{~mm}$ est à rapprocher du rayon de courbure de $14 \mathrm{~mm}$, et l'engagement de $12 \mathrm{~mm}$ du rayon de $8 \mathrm{~mm}$. Pour les deux valeurs choisies, il y a une bonne corrélation entre engagement radial réel rectiligne, et en courbure. Les disparités observées sur la zone A de la figure 7 et les zones A et B de la figure 8 sont probablement à mettre sur le compte des 


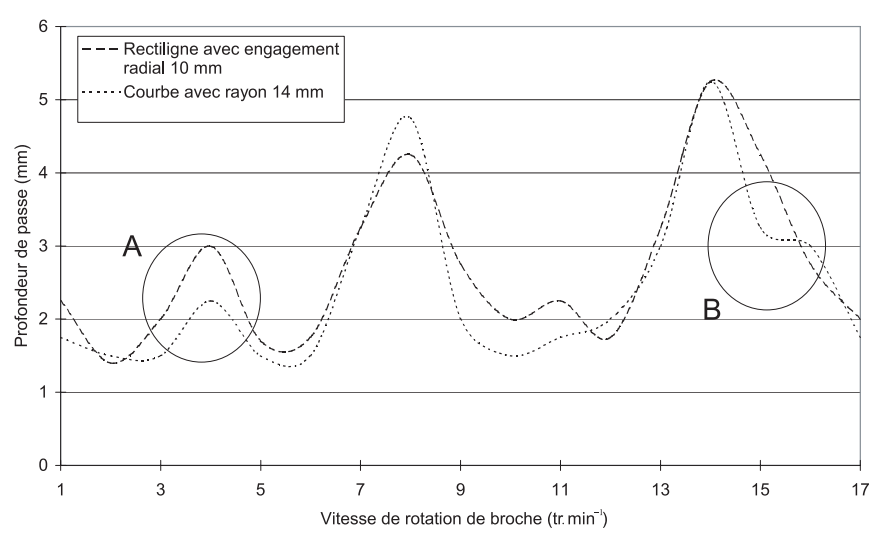

Fig. 8. Comparaison des lobes de stabilité pour une trajectoire rectiligne d'engagement radial de $10 \mathrm{~mm}$ et un rayon de courbure de $14 \mathrm{~mm}$.

imprécisions liées à l'expérimentation et à la mesure. La variation d'engagement radial réel est, compte tenu des réglages faits précédemment, le phénomène prépondérant conduisant à l'apparition de la vibration dans les trajectoires courbes en usinage de poche.

\section{Conclusion}

Si l'usinage grande vitesse permet des gains d'enlèvement de matière importants, ils peuvent être cependant limités par l'apparition de vibrations pendant l'usinage. Les diagrammes de lobes de stabilité offrent une carte de la limite de stabilité pour des paires vitesse de rotation de broche/profondeur de passe. La plupart du temps ces diagrammes, théoriques ou expérimentaux, sont construits pour rendre compte du comportement vibratoire d'usinages rectilignes. Ils peuvent être utilisés pour des usinages contenant des portions courbes, comme en usinage de poche à fond plat, en appliquant certaines règles.

Le passage de rayons de courbure concaves a tendance à être plus instable que les trajectoires rectilignes, les rayons convexes sont eux plus stables donc ne présentent pas de risque de vibration. Pour se rapprocher du comportement en trajectoire linéaire pour ces rayons convexes, il faut tout d'abord choisir une certain nombre d'options de programmation de la commande numérique. Tous ces choix vont dans la même direction : garder la vitesse d'avance réelle du mobile pendant l'usinage la plus proche de la consigne. Les diagrammes des lobes de stabilité obtenus dans ces conditions pour différents rayons de courbure montrent un affaissement et un décalage des lobes vers des profondeurs de passe plus faibles. Ce décalage correspond majoritairement, dans les conditions citées cidessus, à la variation de l'engagement radial réel. Une maîtrise de cet engagement le long de la trajectoire, ou à défaut, une connaissance de l'engagement radial réel et de la limite de stabilité lui correspondant en trajectoire rectiligne, permet de réutiliser les lobes de stabilité en trajet rectiligne pour tout type de poche à fond plat.

Remerciements. Nous tenons à remercier pour leur collaboration active à divers stades de cette étude Mlle Valérie Pinault, M. Julien Gravet et M. Vincent Pateloup pour son apport sur l'évaluation de l'engagement radial réel.

\section{Références}

[1] S. Coromant, Comment réduire les vibrations lors de l'usinage des métaux, Guide de l'utilisateur Sandvik Coromant 1997.9, 1997, pp. 44-58

[2] G. Peigne, Étude et simulation des effets dynamiques de la coupe sur la stabilité de la coupe et la qualité géométrique de la surface usinée : application au fraisage de profil, Thèse de l'Institut national polytechnique de Grenoble, 2003

[3] Y. Altintas, Manufacturing Automation, Cambridge University Press, 2000

[4] S. Engin, Y. Altintas, Mechanics and dynamics of general milling cutter, Part I : helical end mills, Int. J. Machine Tools and Manufacture, Pergamon, 41 (2001) 2195-2212

[5] R.P.H. Faassen, N. Van de Wouw, Prediction of regenerative chatter by modelling and analysis of high-speed milling, Int. J. Machine Tools and Manufacture, Pergamon, 43 (2003) 1437-1446

[6] S. Assouline, E. Beauchesne, Simulation numérique de l'usinage à l'échelle macroscopique : modèles dynamiques de la pièce, Mécanique \& Industries 3 (2002) 389-402

[7] T. Insperger, G. Stepan, Multiple chatter frequencies in milling processes, J. Sound and Vibration 262 (2003) 333-345

[8] P.V. Bayly, B.P. Mann, Effects of radial immersion and cutting direction on chatter instability in end-milling, Proceedings of IMECE2002, ASME, 2002

[9] T.L. Schmitz, Automatic trimming of machining stability lobes, Int. J. Machine Tools and Manufacture, Pergamon, 42 (2002) 1479-1486

[10] S. Bloch, E. Deneuville, L. Tan, Innovative Feed Rate Optimisation Technique, 3rd international congress on metal cutting and high speed milling, Metz, 2001

[11] Siemens, Manuel de programmation Sinumerik 840D Notions de base, Edition 04-2000, 1999, pp. 5.157-5.172 